\title{
Survival outcomes and toxicity of intraoperative intraperitoneal chemotherapy in advanced epithelial ovarian cancer
}

\author{
Ji-Young Yoon, Yu-Jin Koo, Mi-Jung Kim, Tae-Jin Kim, Kyung-Taek Lim, Ki-Heon Lee \\ Department of Obstetrics and Gynecology, Cheil General Hospital and Women's Healthcare Center, Kwandong University College of Medicine, Seoul, Korea
}

\section{Objective}

To assess the effect of single-dose cisplatin intraperitoneally administered during cytoreductive surgery in advanced epithelial ovarian cancer.

\section{Methods}

Data from patients who underwent surgical management followed by intravenous (IV) chemotherapy for stage III epithelial ovarian cancer from 2003 to 2012 were retrospectively reviewed. Subjects were divided into intraperitoneal (IP) and no-intraperitoneal (NIP) groups according to the administration of IP cisplatin $100 \mathrm{mg}$ during the staging surgery. Clinical results such as survival outcomes and chemotherapeutic toxicity were compared between the two groups.

\section{Results}

Thirty-seven patients in the IP group and 26 in the NIP group were identified. There were no significant differences between the two groups in basic characteristics such as age, histology, and surgical procedures. After the surgery with or without IP chemotherapy, there was no difference in the rate of either hematologic or gastrointestinal toxicity or in the rate of incompletion of following IV chemotherapy. Tumor recurrence occurred in $67.6 \%$ (25 patients) of IP group and $57.7 \%$ (15 patients) of NIP group $(P=0.423)$ during the mean follow-up period of 37 months. The 3-year disease free-survival rate was $39.9 \%$ in the IP group and $35.8 \%$ in the NIP group, and the relative risk of recurrence was 0.864 (95\% confidence interval, $0.447-1.673 ; P=0.665)$ in the IP group as compared with the NIP group.

\section{Conclusion}

IP chemotherapy with single-dose cisplatin during cytoreductive surgery is safe and feasible with little chemotherapeutic toxicity in advanced epithelial ovarian cancer, but no distinct improvement in survival could be demonstrated in the present study.

Keywords: Intraperitoneal chemotherapy; Ovarian neoplasms; Survival outcome; Toxicity

\section{Introduction}

Epithelial ovarian cancer (EOC) is the leading cause of death in women with gynecologic malignancies worldwide. In 2013, there were an estimated 22,240 new cases of ovarian cancer and 14,030 deaths in the United States [1]. In Korea, 2,215 patients were newly diagnosed with ovarian cancer between 1993 and 1995, and the number increased steadily to 8,391 between 2006 and 2010 [2]. EOC is rarely diagnosed during the early stages because there is still no effective screening tool and the symptoms are mostly unspecific as well as late
Received: 2014.4.14. Revised: 2014.6.14. Accepted: 2014.7.15. Corresponding author: Ki-Heon Lee

Department of Obstetrics and Gynecology, Cheil General Hospital and Women's Healthcare Center, Kwandong University College of Medicine, 17 Seoae-ro 1-gil, Jung-gu, Seoul 100-380, Korea Tel: +82-2-2000-7196 Fax: +82-2-2000-7183

E-mail: 1103khl@hanmail.net

Articles published in Obstet Gynecol Sci are open-access, distributed under the terms of the Creative Commons Attribution Non-Commercial License (http://creativecommons. org/licenses/by-nc/3.0/) which permits unrestricted non-commercial use, distribution, and reproduction in any medium, provided the original work is properly cited.

Copyright $\odot 2014$ Korean Society of Obstetrics and Gynecology 


\section{Obstetrics \& Gynecology Science}

Ji-Young Yoon, et al. Intraperitoneal chemotherapy in EOC

developing. Therefore, patients with EOC usually visit the hospital due to a palpable mass in the abdomen or symptoms of tumor metastasis.

A standard treatment of EOC is maximal cytoreductive surgery followed by platinum-based intravascular chemotherapy. Over the last several decades, numerous attempts have been made to assess the effect of intraperitoneal (IP) chemotherapy compared to intravenous (IV) chemotherapy. The rationale for IP chemotherapy stems from the speculation that IP administration may be more effective against post-operative residual tumors and initial recurrences which generally remain in the peritoneal cavity [3].

IP chemotherapy was first tried in 1978 [4], and three recent randomized trials reported a significantly improved survival for IP over IV chemotherapy in patients with EOC [5-7]. In the Gynecologic Oncology Group (GOG) 172 trial [5], a milestone study investigating the survival between the two groups, patients with stage III ovarian or primary peritoneal cancer were randomized to receive IV paclitaxel and cisplatin versus IV paclitaxel, followed by IP cisplatin and paclitaxel. There was significant prolongation of both progression-free survival (PFS, 24 vs. 18 months) and overall survival (OS, 66 vs. 50 months) in the IP group compared to the IV group. Finally, in 2006, the National Cancer Institute in the United States issued a clinical statement suggesting that patients with stage III EOC who had received optimal debulking surgery should be considered for IP chemotherapy [8].

However, in spite of the compelling results of the study, several issues still remain unclear; specifically, it is unknown whether any protocol of IP administration can be helpful for survival and which chemotherapeutic regimen is optimal. In addition, several studies indicated that IP chemotherapy was associated with a higher rate of grade III or IV toxicity, particularly in terms of gastrointestinal disorders such as abdominal pain, ileus, nausea, vomiting, and diarrhea [5], because of direct contact between chemotherapeutic drugs and the peritoneum or bowel. Limitations of IP chemotherapy include not only the high toxicity profile and consequent low completion rate, but also inconvenient administration using an IP catheter. Several researchers have therefore introduced new IP chemotherapy techniques and various protocols modified from GOG 172 [9]. In this study we aimed to assess the efficacy of singledose IP cisplatin administered immediately after completion of cytoreductive surgery in advanced stage of EOC, focusing on the effect on survival and chemotherapeutic toxicity.

\section{Materials and methods}

A retrospective review was performed to identify patients treated by surgical debulking followed by IV chemotherapy for EOC from February 2003 to October 2012 at Cheil General Hospital and Women's Healthcare Center. The analysis was restricted to newly diagnosed disease of stage III according to the International Federation of Gynecology and Obstetrics (FIGO), following cytoreductive surgery. The surgical procedures involved total hysterectomy, bilateral salpingooophorectomy, peritoneal cytology, omentectomy, multiple biopsies of peritoneal and pelvic peritoneum, and bilateral pelvic lymph node dissection with or without para-aortic lymph node dissection. An appendectomy was performed in cases of mucinous histology. An effort was made to achieve maximal cytoreduction to less than $1 \mathrm{~cm}$ residual disease or resection of all visible disease, if possible.

IP chemotherapy was provided according to the clinician's preference. Surgery was conducted via either a laparoscopic or abdominal approach, but IP chemotherapy was limited only to cases of laparotomy due to the convenience of administration. At the end of surgery, cisplatin $\left(100 \mathrm{mg} / \mathrm{m}^{2}\right)$ was diluted in $300 \mathrm{~mL}$ of normal saline and administered into the abdominal cavity by a semi-closed technique, in which the drug was injected into the abdominal cavity using a standard manual syringe immediately before the abdominal wall was completely closed. A Jackson-Pratt drain was placed into abdominal cavity and remained locked for 24 hours after surgery to maintain the concentration of IP cisplatin.

After cytoreductive surgery, all patients were treated with IV chemotherapy regardless of IP chemotherapy. After postoperative recovery, they were scheduled to receive a combination agent consisting of paclitaxel $\left(175 \mathrm{mg} / \mathrm{m}^{2}\right)$ IV and carboplatin (area-under-the-curve of 5) IV for three to six cycles every three weeks. Anti-emetic prophylaxis with ramosetron hydrochloride $(0.3 \mathrm{mg}) \mathrm{IV}$, dexamethasone $(20 \mathrm{mg}$ at 12 and 6 hours prior to treatment) IV, and cimetidine (300 mg) IV was provided prior to chemotherapy. Pre-hydration with a liter of normal saline was also instituted 6 hours prior to paclitaxel administration. Immediately following IV combination chemotherapy, administration of an additional $2 \mathrm{~L}$ of $5 \%$ dextrose saline was initiated. A regular evaluation with clinical assessment and measurement of absolute neutrophil count, platelet count, liver enzyme, renal function, and serum CA-125 was performed the day before chemotherapy, once every 3 weeks.

All toxicities were graded according to the Common Toxicity 


\title{
Obstetrics \& Gynecology Science
}

\author{
Vol. 57, No. 6, 2014
}

Criteria for Adverse Events ver. 4.0. Patients with greater than grade 1 neutropenia were treated with granulocyte colony stimulating factor to achieve an absolute neutrophil count $\geq 1.5 \times 10^{9} / \mathrm{L}$. IV chemotherapy was discontinued when initial modifications, such as dose reduction or cycle delays for up to 2 weeks, failed to improve drug-related toxicity. Completion was defined by receipt of all courses planned before starting chemotherapy infusion.

During chemotherapy, positron emission tomography/computerized tomography (PET/CT) imaging was used every three cycles of chemotherapy to estimate disease progression. After completing the initial treatment, routine follow-up comprising a clinical examination and CA-125 level check were performed every 3 months for the next 2 years and every 3 to 6 months for the following 3 years. A PET/CT scan was performed every 6 to 12 months for 5 years or when clinically indicated.

Demographic characteristics, pathology reports, and progressive courses related to post-chemotherapy complications and disease progression were obtained from medical records. In order to evaluate the implications of IP chemotherapy, patients were divided into two groups; IP group and nointraperitoneal (NIP) group according to the administration of intraoperative IP cisplatin. Statistical analyses were performed using the IBM SPSS ver. 20 (IBM Corp., Armonk, NY,
USA). Continuous variables were compared between the two groups using Student's t-test, and categorical variables were compared using a two-tailed chi-square test, as appropriate. Estimates of the cumulative proportions of survival were based on the Kaplan-Meier procedure. Estimates of the relative risk and confidence intervals for treatment effects with respect to recurrence and death were generated with use of the Cox model. Surviving patients were censored at the date of last follow-up. Differences were considered statistically significant if $P<0.05$.

\section{Results}

A total of 63 patients who met the inclusion criteria were identified during the study period. Twenty-six patients were treated with only IV chemotherapy while the other 37 patients received both IP chemotherapy during surgery and adjuvant IV chemotherapy. There were no significant differences between the two groups regarding parity, body mass index, previous surgical history, and initial CA-125 and hemoglobin levels (Table 1). The mean age of patients was 54 and 53 years in the IP and NIP group, respectively $(P=0.689)$, and all but two of the patients underwent abdominal surgery in entire group. Tumor

Table 1. Demographic characteristics of the patients who underwent surgical management followed by intravenous chemotherapy with or without IP chemotherapy for stage III epithelial ovarian cancer

\begin{tabular}{|c|c|c|c|}
\hline & IP group $(n=37)$ & NIP group $(n=26)$ & $P$-value \\
\hline Age (yr) & $54.2 \pm 10.8$ & $53.1 \pm 11.4$ & 0.689 \\
\hline Gravida & $3.3 \pm 2.2$ & $3.4 \pm 2.4$ & 0.933 \\
\hline Parity & $1.9 \pm 1.2$ & $1.9 \pm 1.5$ & 0.918 \\
\hline Body mass index $\left(\mathrm{kg} / \mathrm{m}^{2}\right)$ & $23.7 \pm 3.3$ & $22.9 \pm 2.7$ & 0.351 \\
\hline Previous history of abdomino-pelvic surgery & & & 0.879 \\
\hline None & $23(62.2 \%)$ & $17(65.4 \%)$ & \\
\hline Laparoscopy & $1(2.7 \%)$ & 0 & \\
\hline Laparotomy & $13(35.1 \%)$ & $9(34.6 \%)$ & \\
\hline Preoperative CA-125 (U/mL) & $1,667.6 \pm 515.2$ & $1,886.3 \pm 600.4$ & 0.783 \\
\hline Tumor size $(\mathrm{cm})$ & $8.9 \pm 3.3$ & $11.8 \pm 6.6$ & 0.045 \\
\hline Surgical mode & & & 0.166 \\
\hline Laparotomy & $37(100 \%)$ & $24(92.3 \%)$ & \\
\hline Laparoscopy & 0 & $2(7.7 \%)$ & \\
\hline Hemoglobin on postoperative day 3 (mg/mL) & $9.4 \pm 1.6$ & $9.4 \pm 1.7$ & 0.983 \\
\hline
\end{tabular}

Data are shown as mean \pm standard deviation or number of patients (\%).

IP, intraperitoneal; NIP, no-intraperitoneal. 


\section{Obstetrics \& Gynecology Science}

Ji-Young Yoon, et al. Intraperitoneal chemotherapy in EOC

size was slightly larger in the NIP than in the IP group (11.8 vs. $8.9 \mathrm{~cm}, P=0.045)$.

In surgical procedures, para-aortic lymphadenectomy was performed in 31 (83.8\%) patients of the IP group and 21 patients $(80.8 \%)$ of the NIP group $(P=0.75)$ (Table 2$)$. The rate of patients with residual tumor greater than $1 \mathrm{~cm}$ was also similar between the groups, with $8.1 \%$ (3 patients) in the IP group and $11.5 \%$ (3 patients) in the NIP group $(P=0.684)$. The rate of FIGO stage IIIa, IIIb, and IIIc was $5.4 \%, 10.8 \%$, and $83.8 \%$ in the IP group, and $0 \%, 3.8 \%$, and $96.2 \%$ in the NIP

Table 2. Surgical and pathologic outcomes

\begin{tabular}{|c|c|c|c|}
\hline & IP group $(n=37)$ & NIP group $(n=26)$ & $P$-value \\
\hline \multicolumn{4}{|c|}{ Surgical procedures } \\
\hline PALND & $31(83.8 \%)$ & $21(80.8 \%)$ & 0.75 \\
\hline Residual disease & & & 0.684 \\
\hline No & $34(91.9 \%)$ & $23(88.5 \%)$ & \\
\hline Yes (>1 cm) & $3(8.1 \%)$ & $3(11.5 \%)$ & \\
\hline FIGO stage & & & 0.11 \\
\hline Illa & $2(5.4 \%)$ & 0 & \\
\hline Illb & $4(10.8 \%)$ & $1(3.8 \%)$ & \\
\hline IIIC & $31(83.8 \%)$ & $25(96.2 \%)$ & \\
\hline Histology & & & 0.814 \\
\hline Serous papillary & $25(67.6 \%)$ & $17(65.4 \%)$ & \\
\hline Clear cell & $5(13.5 \%)$ & $5(19.2 \%)$ & \\
\hline Mucinous & 0 & $2(7.7 \%)$ & \\
\hline Endometrioid & $2(5.4 \%)$ & 0 & \\
\hline Other & $5(13.5 \%)$ & $2(7.7 \%)$ & \\
\hline Grade & & & 0.398 \\
\hline 1 & $2(5.4 \%)$ & $3(11.5 \%)$ & \\
\hline 2 & $4(10.8 \%)$ & $3(11.5 \%)$ & \\
\hline 3 & $31(83.8 \%)$ & $20(76.9 \%)$ & \\
\hline
\end{tabular}

Data are shown as number of patients (\%).

IP, intraperitoneal; NIP, no-intraperitoneal; PALND, para-aortic lymph node dissection.

Table 3. Time interval and cycle number of IV chemotherapy and clinical progress

\begin{tabular}{|c|c|c|c|}
\hline & IP group $(n=37)$ & NIP group $(n=26)$ & $P$-value \\
\hline Time to start of IV chemotherapy after surgery (day) & $14.9 \pm 4.4$ & $15.6 \pm 6.2$ & 0.597 \\
\hline Mean number of the first-line IV chemotherapy cycles & $6.5 \pm 1.6$ & $5.9 \pm 1.2$ & 0.068 \\
\hline Tumor recurrence & $25(67.6)$ & $15(57.7)$ & 0.423 \\
\hline $\begin{array}{l}\text { Mean number of total IV chemotherapy cycles during the } \\
\text { total follow-up period }\end{array}$ & $15.7 \pm 9.9$ & $11.0 \pm 7.0$ & 0.042 \\
\hline Current status & & & 0.148 \\
\hline No evidence of disease & $19(51.4)$ & $16(61.5)$ & \\
\hline Alive with disease & $6(16.2)$ & $7(26.9)$ & \\
\hline Death of disease & $12(32.4)$ & $3(11.5)$ & \\
\hline
\end{tabular}

Data are shown as mean \pm standard deviation or number of patients (\%).

IV, intravenous; IP, intraperitoneal; NIP, no-intraperitoneal. 


\title{
Obstetrics \& Gynecology Science
}

\author{
Vol. 57, No. 6, 2014
}

group, respectively, without a statistically significant difference $(P=0.11)$. In both groups, the most common histologic subtype was serous papillary adenocarcinoma $(67.6 \%$ in the IP and $65.4 \%$ in the NIP group), followed by clear cell carcinoma, with a majority of high-grade cases.

The mean time to the start of IV chemotherapy after surgery was 14.9 days in the IP group and 15.6 days in the NIP group $(P=0.597)$, and the mean number of first-line IV chemotherapy was also similar between the two groups (6.5 vs. 5.9 cycles, $P=0.068$ ) (Table 3).

The median duration of follow-up was 32 months (range, 8-123 months) in the IP group and 26 months (range, 2-60 months) in the NIP group. In the IP group, 25 (67.7\%) out of 37 patients relapsed after a median time of 14 months (range, 2-100 months) while in the NIP group, 15 (57.7\%) out of 26 patients relapsed after a median time of seven months (range, 2-25 months). The 3-year disease free-survival (DFS) rate was $39.9 \%$ in the IP group and $35.8 \%$ in the NIP group, and the 3-year OS rate was $78.5 \%$ in the IP group and $79.2 \%$ in the NIP group (Fig 1). The median DFS was 21.0 months in both the IP and NIP groups ( $P=0.659)$. The median OS was 77.0 months in the IP group and it was not reached in the NIP group ( $P=0.435)$. The relative risk of recurrence and death was 0.864 (95\% confidence intervals [Cl], 0.447-1.673; $P=0.665$ ) and $1.673(95 \% \mathrm{Cl}, 0.452-6.188 ; P=0.441)$, respectively, in the IP group as compared with the NIP group. At the time of the present analysis, 19 (51.4\%) patients in the IP group and $16(61.5 \%)$ of the NIP group had no evidence of disease, and $12(32.4 \%)$ patients and three (11.5\%) patients died of disease, respectively $(P=0.148)$.

During the pre-IV chemotherapy examination, grade 2 or 3 anemia was observed more often in the cases of IP chemotherapy $(40.5 \%)$ than in the NIP cases $(23.1 \%)$, but the difference was not statistically significant $(P=0.103)$ (Table 4). Similarly, the rate of any grade of nausea or vomiting was higher in the IP group (21.6\%) than in the NIP group (8.4\%) without a significant difference $(P=0.287)$. It is notable that none of the patients who received IP chemotherapy experienced any grade of neutropenia or thrombocytopenia. During the firstline IV chemotherapy, hematotoxicities and gastrointestinal complications such as nausea, vomiting, abdominal pain, diarrhea, and ileus were similar between the two groups.

Twelve (32.4\%) patients of the IP group and nine (34.6\%) patients of the NIP group experienced either discontinuation or cycle modification such as cycle delay or dose reduction in the first-line IV chemotherapy, but the rate was not significantly different between the groups $(P=0.856)$. The main reasons for these changes were neutropenia grade 2 or higher (16 cases), or elevated liver-enzyme (3 cases), anemia (1 case), or thrombocytopenia (1 case).

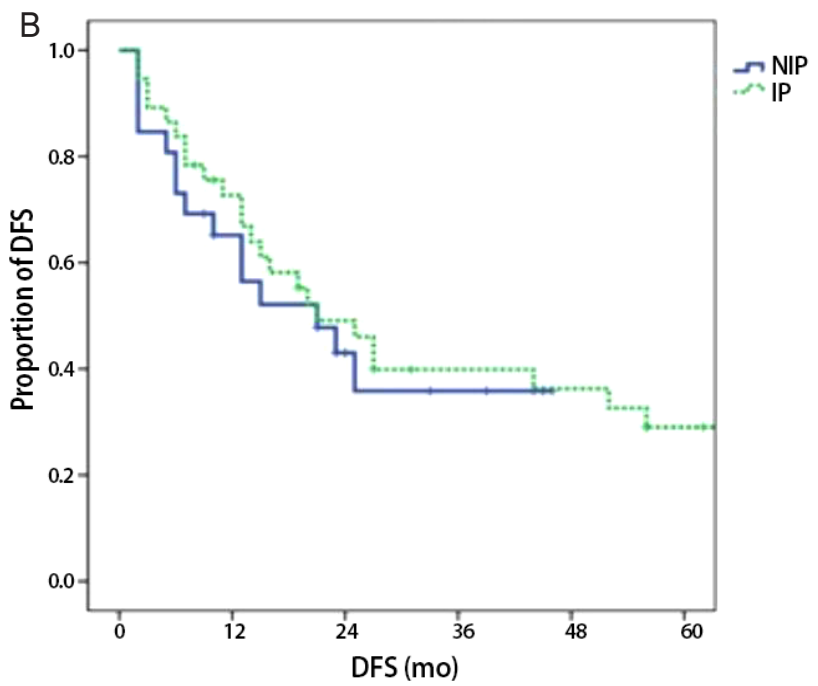

Fig. 1. Kaplan-Meier analysis shows disease-free survival (DFS) and overall survival (OS) among the 63 eligible patients with stage III epithelial ovarian cancer who were treated with cytoreductive surgery followed by intravenous chemotherapy with or without intraperitoneal (IP) chemotherapy. The 3-year DFS (A) was 39.9\% in the IP group and 35.8\% in the no-intraperitoneal (NIP) group, and the 3-year OS (B) was $78.5 \%$ in the IP group and $79.2 \%$ in the NIP group. The median DFS was 21.0 months in both the IP and NIP groups (P=0.659). The median OS was 77.0 months in the IP group and it was not reached in the NIP group $(P=0.435)$. 


\section{Obstetrics \& Gynecology Science}

Ji-Young Yoon, et al. Intraperitoneal chemotherapy in EOC

\section{Discussion}

Our findings demonstrated that IP chemotherapy with singledose cisplatin administered immediately after completion of cytoreductive surgery in advanced EOC had no distinct effect on the prolongation of survival, although it is safe and feasible with a low toxicity.

In 2011, a Cochran meta-analysis [10] was conducted on

Table 4. Chemotherapy related toxicities before and during first-line IV chemotherapy

\begin{tabular}{|c|c|c|c|}
\hline & IP group $(n=37)$ & NIP group $(n=26)$ & $P$-value \\
\hline \multicolumn{4}{|l|}{ Before first-line IV chemotherapy } \\
\hline Anemia & & & 0.103 \\
\hline Grade 1 & $22(59.5)$ & $20(76.9)$ & \\
\hline Grade 2 & $13(35.1)$ & $6(23.1)$ & \\
\hline Grade 3 & $2(5.4)$ & 0 & \\
\hline Neutropenia $\left(\right.$ ANC $\left.<1,500 / \mathrm{mm}^{3}\right)$ & 0 & 0 & \\
\hline Thrombocytopenia $\left(<100,000 / \mathrm{mm}^{3}\right)$ & 0 & 0 & \\
\hline Nausea/vomiting & & & 0.287 \\
\hline None & $29(78.4)$ & $22(91.7)$ & \\
\hline Grade 1 & $6(16.2)$ & $1(4.2)$ & \\
\hline Grade 2 & $2(5.4)$ & $1(4.2)$ & \\
\hline Abdominal pain & & & 0.862 \\
\hline None & $33(89.2)$ & $22(91.7)$ & \\
\hline Grade 1 & $4(10.8)$ & $1(4.2)$ & \\
\hline Grade 2 & 0 & $1(4.2)$ & \\
\hline Diarrhea & & & 0.66 \\
\hline None & $35(94.6)$ & $23(95.8)$ & \\
\hline Grade 1 & $2(5.4)$ & $1(4.2)$ & \\
\hline lleus & & & 1.0 \\
\hline None & $35(94.6)$ & $23(95.8)$ & \\
\hline Grade 1 & 0 & 0 & \\
\hline Grade 2 & $2(5.4)$ & $1(4.2)$ & \\
\hline \multicolumn{4}{|l|}{ During first-line IV chemotherapy } \\
\hline Anemia & & & 0.371 \\
\hline Grade 1 & $19(51.4)$ & $17(65.4)$ & \\
\hline Grade 2 & $17(45.9)$ & $8(30.8)$ & \\
\hline Grade 3 & $1(2.7)$ & $1(3.8)$ & \\
\hline Neutropenia & & & 0.404 \\
\hline Grade 1 & $23(62.2)$ & $16(61.5)$ & \\
\hline Grade 2 & $8(21.6)$ & $2(7.7)$ & \\
\hline Grade 3 & $4(10.8)$ & $5(19.2)$ & \\
\hline Grade 4 & $2(5.4)$ & $3(11.5)$ & \\
\hline Thrombocytopenia $\left(<100,000 / \mathrm{mm}^{3}\right)$ & $1(2.7)$ & 0 & 1.0 \\
\hline Discontinuation or cycle modification of the first-line IV & $12^{a)}(32.4)$ & $9^{\text {b) }}(34.6)$ & 0.856 \\
\hline chemotherapy & & & \\
\hline
\end{tabular}

Based on the Common Toxicity Criteria for Adverse Events ver. 4.0; Data are shown as number (\%).

IV, intravenous; IP, intraperitoneal; NIP, no-intraperitoneal; ANC, absolute neutrophil count.

${ }^{a}$ Due to neutropenia G2 or higher in 9 patients, high liver-enzyme in one, anemia in one, and thrombocytopenia in one; b) Due to neutropenia G2 or higher in 7 and high liver-enzyme in two. 


\title{
Obstetrics \& Gynecology Science
}

\author{
Vol. 57, No. 6, 2014
}

2,119 women from nine randomized trials, including three randomized phase 3 GOG trials. The study concluded that if patients with EOC underwent chemotherapy with an IP component, they were less likely to die (hazard ratio [HR], 0.81; $95 \% \mathrm{Cl}, 0.72-0.90)$, as well as less likely to experience tumor recurrence (HR, $0.78 ; 95 \% \mathrm{Cl}, 0.70-0.86)$. However, all of these trials adopted the repeated protocol for IP chemotherapy, which was performed at three to four week intervals for a total of at least six cycles. As a result, there was greater serious toxicity with regard to gastrointestinal effects, pain, fever, and infection, and thus a lower rate of women who received all assigned chemotherapy by the IP compared to the IV route.

In the high-quality GOG 172 trail, one of the chemotherapy regimens consisted of $135 \mathrm{mg} / \mathrm{m}^{2}$ of IV paclitaxel over a 24hour period on day 1 , followed by $100 \mathrm{mg} / \mathrm{m}^{2}$ of IP cisplatin on day 2 and $60 \mathrm{mg} / \mathrm{m}^{2}$ of IP paclitaxel on day 8 , every 3 weeks. Despite the notable improvement in survival, only $42 \%$ of the patients randomized to the IP arm completed 6 cycles of chemotherapy, while $83 \%$ of the patients in the IV arm did. Leukopenia (76\%) was the most common severe (grades 3 or 4) complication, and other hematologic events, except for leukopenia and thrombocytopenia, occurred in $94 \%$ of the patients with IP treatment. Analysis of the GOG 172 trial led Walker et al. [11] to conclude that it was difficult to deliver six cycles of IP therapy without complications. Multiple reasons for discontinuing IP therapy were classified according to their relationship to the peritoneal access device, and clearly or possibly catheter-related issues accounted for as much as $66.4 \%$ of discontinuations.

In this regard, it is important to consider administration of single-dose IP chemotherapy immediately after the surgery, such as hyperthermic intraperitoneal chemotherapy (HIPEC). HIPEC is a locoregional treatment which allows microscopic cytoreduction by combining the regional pharmacokinetic advantage of IP drug delivery and the synergistic enhancement of drug cytotoxicity induced by heat [12]. After reaching an intra-abdominal temperature of at least $41^{\circ} \mathrm{C}$, a perfusate containing chemotherapy agents (mitomycin $\mathrm{C}$ plus cisplatin or doxorubicin plus cisplatin) is injected into the abdomen and quickly drained through the temporary catheters. In this way, HIPEC has the advantage of not only IP therapy, suggesting high target to plasma drug concentration ratios and prolonged serum exposures, but also hyperthermia, providing increased cellular metabolism and permeability without placing catheters for repeated injection.

However, since HIPEC also has several limitations, namely high cost, prolonged operation time, heat-related risks, and additional equipment such as a pump or heat exchanger, single IP chemotherapy without a heating procedure has been reported in a few small studies. Most recently, Kim et al. [13] evaluated the feasibility of a cisplatin dose of $70 \mathrm{mg} / \mathrm{m} 2$ in $1 \mathrm{~L}$ normal saline administered intraperitoneally for 24 hours after cytoreductive surgery in patients with stage IC to IV of EOC. They concluded that the intraoperative IP chemotherapy was feasible and safe, because most of the adverse events (87\%) were grade 1 or 2 , and none of the gastrointestinal events were grade 3 or more. Grade 3 events were all anemia, which was mentioned as a surgical complication, rather than IP chemotherapy, although it was unable to be verified. These results are consistent with our data, suggesting that anemia was the only complication of grade 3 or more. In the present study, however, IP and NIP group had the same hemoglobin level on postoperative day 3 , which implies that anemia may be a pure complication of IP chemotherapy, although the difference was not statistically significant.

In the last decade, there have been several reports indicating that HIPEC shows promising results in terms of survival outcome. In the largest study reported by a Korean group [14], HIPEC using carboplatin and interferon-a was performed after primary debulking surgery and IV chemotherapy, and results were compared to those obtained with patients treated with primary debulking and IV chemotherapy. A significant improvement in OS was observed in the HIPEC group. In the study by Kim et al. [13] which had a similar protocol to our study, only ten patients were involved, and there was one case of recurrence during the follow-up period of 4 to 39 months. Besides, it was not a comparison study between IP and IV chemotherapy, but a single-arm analysis of IP therapy, so the survival evaluation lacked the necessary power to determine the efficacy of IP therapy. On the other hand, our study was designed to compare IP and IV chemotherapy versus IV chemotherapy alone in a total of 63 patients. The 3-year DFS was slightly higher in the IP group (39.9\%) than in the NIP group $(35.8 \%)$, and the relative risk of recurrence was 0.864 in the IP group. It was unfortunate, however, that the difference in survival improvement was not statistically significant in spite of the same tumor stage and surgical procedures. There could be multiple reasons for this result, including inadequate dose, single cycle, single regimen, short residence time of intraperitoneal drug, and small sample size.

To the best of our knowledge, this is the first study to evaluate both survival and toxicity of single IP chemotherapy with 


\section{Obstetrics \& Gynecology Science}

Ji-Young Yoon, et al. Intraperitoneal chemotherapy in EOC

unheated cisplatin compared to conventional treatment in primary EOC. We note that our study is inherently limited due to its retrospective nature, and thus a randomized prospective study in a large population still needs to be performed. Secondly, our study had a small sample size, which may have led to the survival difference not being significant. In summary, this study did not provide evidence that intraoperative unheated IP chemotherapy with cisplatin improved survival in advanced EOC. Even so, the protocol still had definite benefits in terms of adverse events, such as no catheter-related complications and less hematologic and non-hematologic toxicities than cyclic IP chemotherapy, which can be one of the requirements for ideal chemotherapy. The survival outcome should be confirmed in further large and prospective studies and future work will be required to determine the best protocol and regimen of single IP chemotherapy that fulfills both efficacy and safety requirements.

\section{Conflict of interest}

No potential conflict of interest relevant to this article was reported.

\section{References}

1. Siegel $R$, Naishadham $D$, Jemal $A$. Cancer statistics, 2013. CA Cancer J Clin 2013;63:11-30.

2. Statistics Korea. Cancer incidence [Internet]. Daejeon: Statistics Korea; 2012 [cited 2013 Jan 10]. Available from: http://kostat.go.kr/portal/korea/index.action.

3. Esselen KM, Rodriguez N, Growdon W, Krasner C, Horowitz NS, Campos S. Patterns of recurrence in advanced epithelial ovarian, fallopian tube and peritoneal cancers treated with intraperitoneal chemotherapy. Gynecol Oncol 2012;127:51-4.

4. Dedrick RL, Myers CE, Bungay PM, DeVita VT Jr. Pharmacokinetic rationale for peritoneal drug administration in the treatment of ovarian cancer. Cancer Treat Rep 1978;62:1-11.

5. Armstrong DK, Bundy B, Wenzel L, Huang HQ, Baergen $\mathrm{R}$, Lele $\mathrm{S}$, et al. Intraperitoneal cisplatin and paclitaxel in ovarian cancer. N Engl J Med 2006;354:34-43.
6. Markman M, Bundy BN, Alberts DS, Fowler JM, ClarkPearson DL, Carson LF, et al. Phase III trial of standarddose intravenous cisplatin plus paclitaxel versus moderately high-dose carboplatin followed by intravenous paclitaxel and intraperitoneal cisplatin in small-volume stage III ovarian carcinoma: an intergroup study of the Gynecologic Oncology Group, Southwestern Oncology Group, and Eastern Cooperative Oncology Group. J Clin Oncol 2001;19:1001-7.

7. Alberts DS, Liu PY, Hannigan EV, O'Toole R, Williams SD, Young JA, et al. Intraperitoneal cisplatin plus intravenous cyclophosphamide versus intravenous cisplatin plus intravenous cyclophosphamide for stage III ovarian cancer. N Engl J Med 1996;335:1950-5.

8. National Cancer Institute. $\mathrm{NCl}$ clinical announcement: intraperitoneal chemotherapy for ovarian cancer [Internet]. Rockville: National Cancer Institute; c2006 [cited 2014 Oct 6]. Available from: http://ctep.cancer.gov/highlights/ docs/clin_annc_010506.pdf.

9. Ramarajapalli ML, Kilara NG, Subramanyam M, Rao NA. Technique of intraperitoneal chemotherapy using veress needle in patients with ovarian cancer. Int J Gynecol Cancer 2011;21:1388-90.

10. Jaaback K, Johnson N. Intraperitoneal chemotherapy for the initial management of primary epithelial ovarian cancer. Cochrane Database Syst Rev 2006;(1):CD005340.

11. Walker JL, Armstrong DK, Huang HQ, Fowler J, Webster K, Burger RA, et al. Intraperitoneal catheter outcomes in a phase III trial of intravenous versus intraperitoneal chemotherapy in optimal stage III ovarian and primary peritoneal cancer: a Gynecologic Oncology Group Study. Gynecol Oncol 2006;100:27-32.

12. Deraco M, Virzi S, lusco DR, Puccio F, Macri A, Famulari $C$, et al. Secondary cytoreductive surgery and hyperthermic intraperitoneal chemotherapy for recurrent epithelial ovarian cancer: a multi-institutional study. BJOG 2012;119:800-9.

13. Kim MJ, Jung YW, Seong SJ, Yoon BS, Kim ML, Joo WD, et al. Intraoperative intraperitoneal chemotherapy with cisplatin in epithelial ovarian cancer. J Gynecol Oncol 2012;23:91-7.

14. Ryu KS, Kim JH, Ko HS, Kim JW, Ahn WS, Park YG, et al. Effects of intraperitoneal hyperthermic chemotherapy in ovarian cancer. Gynecol Oncol 2004;94:325-32. 